\title{
Personalized management of differentiated thyroid cancer in real life - practical guidance from a multidisciplinary panel of experts
}

\author{
Alfredo Campennì $\mathbb{1}^{1} \cdot$ Daniele Barbaro ${ }^{2} \cdot$ Marco Guzzo $^{3} \cdot$ Francesca Capoccetti $^{4} \cdot$ Luca Giovanella $^{5,6}$
}

Received: 28 April 2020 / Accepted: 6 July 2020 / Published online: 9 August 2020

(c) The Author(s) 2020

\begin{abstract}
Purpose The standard of care for differentiated thyroid carcinoma (DTC) includes surgery, risk-adapted postoperative radioiodine therapy (RaIT), individualized thyroid hormone therapy, and follow-up for detection of patients with persistent or recurrent disease. In 2019, the nine Martinique Principles for managing thyroid cancer were developed by the American Thyroid Association, European Association of Nuclear Medicine, Society of Nuclear Medicine and Molecular Imaging, and European Thyroid Association. In this review, we present our clinical practice recommendations with regard to implementing these principles in the diagnosis, treatment, and long-term follow-up of patients with DTC.

Methods A multidisciplinary panel of five thyroid cancer experts addressed the implementation of the Martinique Principles in routine clinical practice based on clinical experience and evidence from the literature.

Results We provide a suggested approach for the assessment and diagnosis of DTC in routine clinical practice, including the use of neck ultrasound, measurement of serum thyroid-stimulating hormone and calcitonin, fine-needle aspiration, cytology, and molecular imaging. Recommendations for the use of surgery (lobectomy vs. total thyroidectomy) and postoperative RaIT are also provided. Long-term follow-up with neck ultrasound and measurement of serum anti-thyroglobulin antibody and basal/stimulated thyroglobulin is standard, with ${ }^{123} /{ }^{131} \mathrm{I}$ radioiodine diagnostic whole-body scans and ${ }^{18} \mathrm{~F}$-fluoro-2deoxyglucose positron emission tomography/computed tomography suggested in selected patients. Management of metastatic DTC should involve a multidisciplinary team.

Conclusions In routine clinical practice, the Martinique Principles should be implemented in order to optimize clinical management/outcomes of patients with DTC.
\end{abstract}

Keywords Differentiated thyroid carcinoma $\cdot$ Martinique Principles $\cdot$ Radioiodine therapy $\cdot$ Recommendations $\cdot$ Surgery

Alfredo Campennì

acampenni@unime.it

1 Department of Biomedical and Dental Sciences and MorphoFunctional Imaging, Nuclear Medicine Unit, University of Messina, Messina, Italy

2 U.O. Endocrinologia, ASL Nord Ovest Toscana, Livorno, Italy

3 Head and Neck Surgery Department, Fondazione IRCCS Istituto Nazionale dei Tumori, Milan, Italy

4 Service Department Macerata Hospital, ASUR Marche AV3, Nuclear Medicine Unit, Macerata, Italy

5 Clinic for Nuclear Medicine and Competence Centre for Thyroid Diseases, Ente Ospedaliero Cantonale, Bellinzona, Switzerland

6 Clinic for Nuclear Medicine, University Hospital and University of Zurich, Zurich, Switzerland

\section{Introduction}

Differentiated thyroid cancer (DTC), most commonly the papillary histotype, accounts for the majority of thyroid cancer cases [1]. The standard of care for DTC includes surgery, risk-adapted postoperative radioiodine therapy (RaIT), and individualized thyroid hormone therapy tailored to the patient's risk of relapse [2]. These approaches lead to excellent responses in $>80 \%$ of patients [2]. However, as DTC carries a significant risk of disease persistence and recurrence, long-term active follow-up is essential [3, 4]. In 2015, the American Thyroid Association (ATA) recommended less intense treatment strategies for many DTC patients, including observation or thyroid lobectomy without RaIT [3], which resulted in controversy and significant differences in clinical practice [5]. In 2019, a joint statement from the ATA, European Association of Nuclear Medicine (EANM), Society of Nuclear Medicine and 
Fig. 1 The Martinique principles summarizing the major points of discussion during the first Martinique meeting. Reproduced with permission from R.M. Tuttle et al. Thyroid (2019) [6]. RaIT radioiodine therapy

\section{Martinique Principles}

1. Advancing our understanding of optimal thyroid cancer management requires a commitment by clinicians, researchers, patients and organizations to engage in proactive purposeful, and inclusive inter-disciplinary cooperation.

2. The goal of RalT should be characterized as remnant ablation, adjuvant treatment, or treatment of known disease using standardized definitions.

3. Assessment of post-operative disease status is required to optimize proper patient selection for RalT (remnant ablation, adjuvant treatment, or treatment of known disease).

4. Post-operative disease status evaluations should be standardized and integrated into routine clinical care.

5. Optimal patient selection for adjuvant RalT requires consideration and evaluation of multiple factors beyond post-operative disease status and risk stratification.

6. The optimal administered activity for adjuvant treatment cannot be definitely determined from the published literature. Until definitive data are available, selection of the administered activity for adjuvant treatment should be based on multidisciplinary management recommendations.

7. Characteristics used to classify patients as RalT-refractory should be used to risk stratify patients with regard to the likelihood that a tumor will response to RalT and not necessarily as definitive criteria to mandate whether or not RalT should be recommended.

8. RalT-refractory criteria will continue to evolve as a) additional studies address important limitations and technical issue confounding the current literature, b) techniques for radioiodine imaging are optimized and standardized, and c) redifferentiation therapies enhance the effectiveness of RalT.

9. Major gaps in knowledge and evidence regarding optimal use of RalT should be addressed with properly designed prospective studies.
Molecular Imaging (SNMMI), and European Thyroid Association (ETA) defined the indications and practical issues of RaIT and agreed on a set of nine principles (the so-called "Martinique Principles"; Fig. 1) [6]. A multidisciplinary panel of five thyroid cancer experts debated the implementation of the Martinique Principles in routine clinical practice, with discussion undertaken via conference call and electronic communication. This paper presents our shared practical recommendations regarding DTC diagnosis, treatment with surgery and RaIT, and long-term follow-up.

\section{Differentiated thyroid cancer diagnosis}

Thyroid nodules are frequently detected in the general population and usually have a low risk of malignancy. When a thyroid nodule is detected (either clinically or incidentally), medical history and physical examination, measurement of thyroid-stimulating hormone (TSH) levels and neck ultrasonography are advised. In addition, measurement of serum calcitonin should be considered to further delineate the possibility of medullary thyroid carcinoma, despite there being a lack of general consensus with regard to routine serum calcitonin measurement [3]. In the presence of reduced or low-normal TSH levels, thyroid scintigraphy is recommended as malignancy is very unlikely in autonomously functioning ("hot") nodules [7]. Notably, although autonomous nodules are almost invariably accompanied by decreased TSH levels (i.e.,
$<0.1-0.4 \mathrm{mUI} / \mathrm{L}$ ) when iodine supply is adequate, the bulk of autonomous tissue may be insufficient to suppress the TSH level in iodine-depleted thyroids, especially in the early phases of autonomy [8]. As a consequence, the decision on whether or not to use thyroid scans should take into account the patient's geographic location and the iodine supply in that area. Independently from the patient's iodine status and the TSH levels, a thyroid scan is also recommended in people with a large multinodular goiter to select suspicious nodules for fine-needle aspiration (FNA), and in people with cytologically indeterminate nodules (i.e., follicular proliferation) to identify a benign compensated functioning adenoma [9].

In nonautonomous nodules, FNA is currently recommended based on ultrasound characteristics. Considerable effort has been made toward the standardization of both ultrasound and FNA cytology reports, with the development of different ultrasound and cytological reporting systems [i.e., TIRADS for ultrasound and the Italian consensus for the classification and reporting of thyroid cytology (SIAPEC)] $[10,11]$. Despite such efforts, up to $25 \%$ of nodules are still considered to be "cytologically indeterminate", requiring in many cases diagnostic surgery to definitely confirm or exclude malignancy (with a prevalence for malignant nodules of about $30 \%$ ). Immunocytochemistry, molecular testing (i.e., mutation analysis, microRNA) $[12,13]$, and imaging techniques, including ${ }^{99 \mathrm{~m}} \mathrm{Tc}$ methoxy-isobutyl-isonitrile (MIBI) scintigraphy and ${ }^{18} \mathrm{~F}$ fluoro-2-deoxyglucose positron emission tomography/ computed tomography ( ${ }^{18}$ F-FDG-PET/CT) [14-17] may be 
Fig. 2 Diagnostic algorithm for patients presenting with thyroid nodules. ${ }^{18} \mathrm{~F}$-FDG-PET/CT ${ }^{18} \mathrm{~F}$ fluoro-2-deoxyglucose positron emission tomography/computed tomography, DTC differentiated thyroid carcinoma, FNA fineneedle aspiration, MIBI methoxy-isobutyl-isonitrile, PEI percutaneous ethanol injection, RAI radioiodine, SIAPEC Italian Societies of Endocrinology and Pathology, TSH thyroid-stimulating hormone

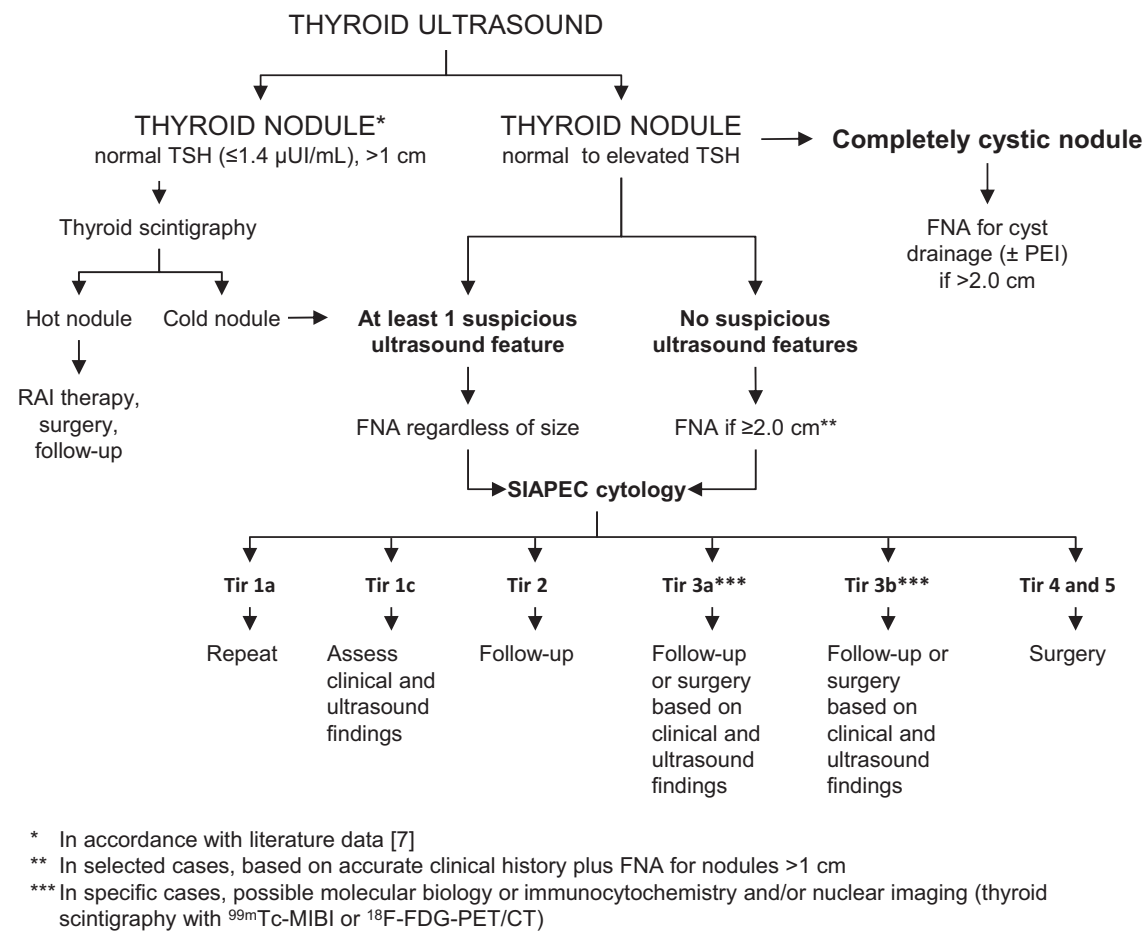

useful in these cases to avoid unnecessary surgeries. Indeed, none of such methods had both near-perfect sensitivity and specificity. Notwithstanding, test performance strongly depends on population-dependent variations in cytology, tumor genetics, and the prevalence of malignancy as well as on the costs and feasibility of the desired diagnostic protocol in the local patient population. Accordingly, the choice of one or more additional test should be a deliberate and multidisciplinary one [18].

Figure 2 summarizes our suggested approach to assessing thyroid nodules in routine clinical practice.

\section{Key points and practical indications}

- Primary diagnostic assessments include neck ultrasound, serum TSH, FNA cytology, and (optional) calcitonin measurement.

- Thyroid scintigraphy (with ${ }^{99 \mathrm{~m}} \mathrm{Tc}$ pertechnetate or ${ }^{123} \mathrm{I}$ ) is recommended when TSH levels are reduced $(<0.3-0.4 \mathrm{mIU} / \mathrm{L})$ and can be considered for TSH levels up to $1.0-1.5 \mathrm{mIU} / \mathrm{L}$ depending on local iodine supply.

- FNA should not be performed in autonomously functioning thyroid nodules confirmed by thyroid scintigraphy, with possible exceptions being clinically or ultrasonographically highly suspicious nodules.

- Using a standardized system to report thyroid ultrasound findings is recommended to provide consistent communication; however, the ultrasonographers' expertize remains pivotal to translate ultrasound results into clinical actions.
- Based on our experience, FNA is suggested when any one of these ultrasound features is present: hypoechoic nodule; nodule with blurred/irregular margins; nodules with microcalcifications $(<2 \mathrm{~mm})$; or nodule that is taller than it is wide.

- In the absence of clearly suspicious ultrasound features, FNA cytology should be considered based on diameter (i.e., $>1-2 \mathrm{~cm}$ ), clinical history, and physical examination.

- Molecular testing and molecular imaging based on ${ }^{99 \mathrm{~m}} \mathrm{Tc}-\mathrm{MIBI}$ scintigraphy and ${ }^{18} \mathrm{~F}-\mathrm{FDG}-\mathrm{PET} / \mathrm{CT}$ may help in refining the diagnosis in selected cases of nodules with indeterminate cytology.

\section{Thyroid surgery}

According to the 2015 ATA guidelines, lobectomy may be sufficient for a unifocal intrathyroidal low-risk carcinoma of $<4 \mathrm{~cm}$ diameter in patients with no prior head and neck radiation, familial thyroid carcinoma, or clinically detectable cervical lymph node metastases, whereas in other cases, total or near total thyroidectomy is recommended [3]. Approximately $20-50 \%$ of patients with DTC have cervical lymph node metastases, mostly in the central neck (i.e., VI Robbins' level) [19]. Central compartment dissection is a well-established treatment of clinical N1a (cN1a) and, coupled with lateral dissection, N1b (cN1b) disease, although its role as prophylaxis in $\mathrm{cNO}$ disease is unclear [3]. Additional factors, including lesion location, 
multifocality, bilaterality, thyroid capsule invasion, and $B R A F^{\mathrm{V} 600 \mathrm{E}}$ and $T E R T$ mutation status, should also be considered to refine surgical planning [20-22].

Overall, we agree with the ATA 2015 recommendations on neck dissection and total thyroidectomy in intermediate- to high-risk DTC; however, the use of lobectomy for low- to intermediate-primary tumors $\leq 4 \mathrm{~cm}$ may be too broad. Current controversies on the extent of surgery probably arise from heterogeneous studies [23]. Notably, the risk for local and distant metastases increases with increasing size of the papillary thyroid cancer (PTC), especially if the tumor size exceeds $2 \mathrm{~cm}$, as has been described in an observational study involving 366 patients with PTC [24].

Moreover, data from the United States National Cancer Data Base showed that patients with papillary DTC sized $2.0-3.9 \mathrm{~cm}$ had improved survival when treated with total thyroidectomy [25]. Accordingly, the Swiss consensus on low-risk papillary DTC limited the definition of low-risk DTC, treatable with lobectomy, to cancer with low-risk ATA 2015 characteristics but with tumor size $<2 \mathrm{~cm}$ [26].

\section{Key points and practical indications}

- More evidence is needed before generalizing the current trend towards use of lobectomy for low-risk primary tumors $\leq 4 \mathrm{~cm}$ in clinical practice. Thus, it may be safer to restrict lobectomy to low-risk tumors $<2 \mathrm{~cm}$.

- In other cases (i.e., low-risk $>2 \mathrm{~cm}$, intermediate- and high-risk DTC), we recommend (near) total thyroidectomy as the first-line procedure, followed by RaIT (discussed below).

- Central compartment dissection is a well-established treatment of cN1a tumors; however, because its role as prophylaxis in $\mathrm{cN} 0$ tumors is uncertain we recommend its use should be avoided in this setting (especially in T1-2 tumors).

- Lateral neck dissection is only indicated in $\mathrm{cN} 1 \mathrm{~b}$ tumors (coupled with central neck dissection).

\section{Postoperative radioiodine therapy}

Following surgery, the risk of structural disease recurrence and/or persistence should be assessed using the three-tier (low, intermediate, and high) stratification suggested by the ATA in 2009 [27] and modified in 2015 [3], while the risk of mortality from thyroid cancer is estimated using the AJCC/TNM staging system [28]. RaIT has long been the standard of care following primary surgery for all patients with DTC, except those with unifocal PTC $<1 \mathrm{~cm}$ and no high-risk characteristics [4]. Based on 2015 ATA guidelines, routine RaIT is recommended for high-risk patients, suggested for intermediate-risk patients, and restricted to selected lowrisk patients [3]. However, Tuttle et al. recognized that the actual goal of RaIT (i.e., ablation of thyroid remnant, adjuvant therapy, and treatment of known disease) can only be determined once the postoperative disease status has been assessed [6]. Then, a comprehensive assessment of risk and postoperative disease status is needed to decide whether RaIT is necessary or if observation will be sufficient. Patients with evidence of persistent disease after appropriate initial surgery may be the only candidates for 'treatment of known disease', regardless of initial risk stratification. In other cases, patients may be candidates for observation, remnant ablation, or adjuvant treatment based on careful risk assessment, as well as patients' preferences. However, there are currently no reliable, universally accepted, precise recommendations to guide the proper assessment of postoperative disease status. Until precise, evidence-based guidelines are available, multidisciplinary teams should establish local standards to guide clinical management that considers the availability and quality of pre- and post-operative imaging and thyroglobulin $(\mathrm{Tg})$ measurements, the experience of operating surgeon, and local clinical concerns [3]. In this setting, it should be considered that whole body scintigraphy (WBS) with single-photon emission computed tomography/CT (SPECT/CT) obtained after administration of therapeutic ${ }^{131}$ I-radioiodine (RAI) activity (i.e., $>1.1 \mathrm{GBq}$ ) and preablation stimulated $\mathrm{Tg}$ measurement remain the most accurate tools for postoperative DTC restaging and are also included in ATA risk stratification system [3].

Accordingly, our panel members agreed on recommending adjuvant RaIT for all patients with high- and intermediate-risk cancer, since it allows for early diagnosis of residual disease and reduces the risk of recurrence [29-32]. In addition, as the risk of local and distant metastases increases with increasing size of the PTC, especially in tumors $>2 \mathrm{~cm}$, the same approach is suggested in low-risk DTC patients with a primary tumor $>2 \mathrm{~cm}$ [26]. Importantly, patients who receive RaIT for ablation or adjuvant purposes with no signs of persistent disease (i.e., undetectable $\mathrm{Tg}$, negative ultrasound and, if performed, negative diagnostic WBS) could be fully reassured and simply monitored every $1-2$ years by clinical examination and basal $\mathrm{Tg}$ measurement avoiding $\mathrm{Tg}$ stimulation tests or periodic neck ultrasound examination during follow-up. Finally, adjuvant treatment is not routinely recommended for low-risk patients with a primary tumor $<2 \mathrm{~cm}$; however, if additional risk factors (i.e., isthmic location, known $B R A F$ mutation) or increased $\mathrm{Tg}$ antibody $(\mathrm{Tg} A b)$ levels are present or a preference for maximum-intensity treatment is expressed by patients, RaIT for remnant ablation is an option. 


\section{Key points and practical indications}

- RaIT has three distinct goals: remnant ablation, adjuvant treatment, or treatment of known residual or recurrent disease.

- For low-risk DTC patients with a primary tumor size $<2 \mathrm{~cm}$ and neither additional risk factors nor elevated $\mathrm{TgAb}$, we recommend observation; routine RaIT is not indicated. Patients who request maximum-intensity treatment, after being informed about their low but existing risk for recurrence, can be treated with RaIT for remnant ablation.

- For patients with low-risk DTC and a primary tumor size of 2-4 cm or additional risk factors, and for patients with intermediate- or high-risk DTC, routine adjuvant RaIT is recommended.

- Pretreatment stimulated Tg and posttreatment Dx-WBSSPECT/CT should always be obtained and serve as platform for restaging and follow-up planning.

\section{Postoperative RalT for remnant ablation or adjuvant treatment}

We generally recommend reducing the daily intake of iodine-containing food for 2 weeks before initiating RaIT [33-36]. Dietary recommendations are listed in Table 1. This is particularly relevant for patients with euthyroid status stimulated with recombinant human TSH (rhTSH) [37]. Measurement of urine iodine content may also be useful, as an association between high urinary iodine excretion and the failure of RaIT has been reported [38].

Conflicting data area reported on RAI activities that should be used for ablative purpose [39-42]. In general, RAI activities of around 1.1-2.2 GBq are used for remnant ablation in selected patients with low-risk DTCs. In these settings, rhTSH use should be preferred considering the better patient quality of life and the lower radiation exposure [39, 43-46].

The optimal RAI activity and stimulation method for adjuvant treatments in patients with intermediate-risk DTC are currently unknown [6]. Accordingly, the use of thyroid hormone withdrawal (THW) or rhTSH and administered RAI activity should be decided on an individual basis in a multidisciplinary setting. Our panel recommends rhTSHstimulated administration of $2.2-3.7 \mathrm{GBq}$ of RAI in intermediate-risk patients. Administration of $\geq 3.7 \mathrm{GBq}$ of RAI is recommended in high-risk patients, preferentially after THW as, according to ATA 2015 guidelines, more controlled data from long-term outcome studies are needed before rhTSH preparation for RAI adjuvant treatment can be recommended for routine practice [3]. However, rhTSH stimulation is mandatory when physiologic TSH
Table 1 Recommendations for reducing iodine intake

\section{SUMMARY}

- No iodized salt

- No dairy products or foods containing dairy products

- No foods from the sea

- Limit grain products (i.e., noodles, pasta, and pastries) to 1 slice of bread, $1 / 2$ cup of pasta daily

- Limit the amount of beef, chicken, and turkey

FOODS TO AVOID

- Iodized salt

- Any vitamins or supplements containing iodine (especially kelp and dulse)

- Milk or other dairy products, including ice cream, cheese, yogurt, and butter

- Seafood, including fish, sushi, shellfish, kelp, and seaweed

- Herbal supplements

- Foods containing the additives carrageen, agar-agar, alginate, or nori

- Commercially prepared bakery products made with iodate dough conditioners

- FD \& $\mathrm{C}$ red dye \#3, which is found in maraschino cherries and occasionally as a pink/red artificial color in beverages

- Egg yolks, whole eggs, and foods containing whole eggs

- Milk chocolate (due to dairy content)

- Blackstrap molasses (unsulfured molasses is fine)

- Soy products (soy sauce, soy milk, tofu) as high soy ingestion has been shown to interfere with RAI uptake

FOODS TO EAT

- Non-iodized salt or non-iodized sea salt

- Egg whites

- Homemade bread made with non-iodized salt and oil (not soybean oil, butter, or milk) or commercially baked breads that do not contain iodate dough conditioners, dairy, or eggs

- Fresh fruits and vegetables

- Frozen vegetables

- Grain, cereal products, and pasta without high iodine ingredients

- Canned fruit

- Natural unsalted nuts and nut butters (e.g., peanut or almond)

- Sodas, beer, wine, lemonade, and fruit juices

- Coffee or tea (without milk, cream, or soy-based nondairy creamer)

- Popcorn popped in vegetable oil or air popped with non-iodized salt

- Black pepper, fresh or dried herbs and spices, and all vegetable oils

- Sugar, jam, jelly, honey, and maple syrup

- Matzoh crackers

POSSIBLE SOURCES OF IODINE INTERFERENCE

- Iodine-containing multivitamins (for 7 days after use)

- Iodine-containing disinfectants, toothpaste, or vaginal lavages (for 2-3 weeks)

- Iodine tincture (for 2-3 weeks)

- Water-soluble iodinated contrast agent (for 3 months) or oil-soluble iodinated contrast agent (for $\geq 3$ months)

- Amiodarone (for 6 months or more if obese)

- Valpressin (for several weeks; suggest testing for ioduria before RaIT) 
Table 1 (continued)

\section{ADDITIONAL GUIDELINES}

- Avoid restaurant foods since there is no reasonable way to determine whether they use iodized or non-iodized salt.

- Consult your doctor before discontinuing any red-colored medication or any iodine-containing medication (e.g., amiodarone, expectorants, or topical antiseptics).

- Avoid all herbal supplements (especially when it is unclear how much iodine they contain).

$R A I$ radioiodine, $R a I T$ radioiodine therapy

stimulation is precluded (e.g., pituitary diseases) or when THW is clinically contraindicated.

Key points and practical indications Postoperative ablation/adjuvant RaIT administration (suggested RAI activities and stimulation protocols):

- Low-risk DTC $(<2 \mathrm{~cm})$ : selective ablation RaIT $(1.1-2.2 \mathrm{GBq})$ with rhTSH stimulation.

- Low-risk $(>2 \mathrm{~cm})$ and intermediate-risk DTC: adjuvant RaIT (2.2-3.7 GBq) with rhTSH stimulation.

- High-risk DTC: adjuvant RaIT ( $\geq 3.7 \mathrm{GBq})$ with THW stimulation.

\section{Long-term follow-up}

Follow-up of DTC after primary treatment has evolved from standard to a more individualized approach that is currently based on ongoing risk assessment [3, 47]. Initial risk assessment is continuously modified and refined by the inclusion of assessments of response to therapy and disease course [3]. Serum $\operatorname{Tg}$ measurements [1, 48, 49], neck ultrasound [50, 51], and ${ }^{123 / 131}$ I-RAI Dx-WBS [51] are commonly used as primary tools for DTC follow-up.

Unstimulated and/or stimulated serum $\mathrm{Tg}$ measurement is currently the yardstick for monitoring patients with DTC after primary therapy. However, its usefulness is limited in patients who are positive for $\mathrm{TgAb}$ since serum $\mathrm{Tg}$ levels can be underestimated when measured using immunometric assays [52, 53].

Neck ultrasound offers several advantages and is a reliable method for detection of loco-regional persistent or recurrent DTC (i.e., thyroid bed and cervical lymph nodes), but is not without limitations [51]. Indeed, the number of neck ultrasounds providing false positive results, which increases the risk of unnecessary and expensive additional procedures, are far from negligible [54-56]. Accordingly, the use of neck ultrasound should be limited (particularly in
Suspected DTC recurrence in patients with increased Tg and negative Dx-WBS

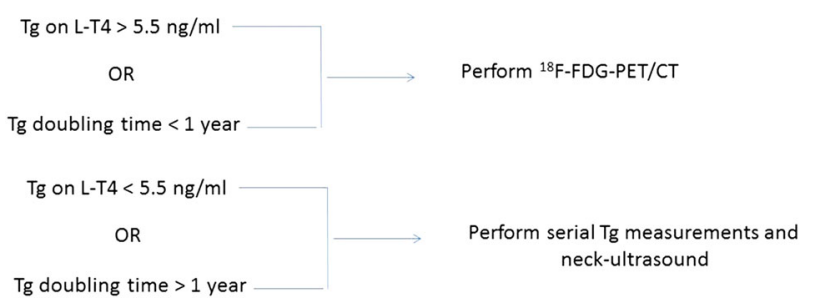

Fig. 3 Assessment of patients with recurrent differentiated thyroid carcinoma (DTC) in relation to thyroglobulin (Tg) level [63]. ${ }^{18}$ FDGPET/CT ${ }^{18}$ F-fluoro-2-deoxyglucose-positron emission tomography/ computed tomography, Dx-WBS diagnostic whole-body scan, Tg-DT thyroglobulin doubling time

low-risk DTC) and, in the absence of $\mathrm{TgAb}$, reserved for patients with unstimulated serum $\mathrm{Tg}$ levels $\geq 1 \mathrm{ng} / \mathrm{mL}$ $[55,57]$.

According to ATA 2015 guidelines, Dx-WBS can be useful for patients with a high- or intermediate-risk of persistent disease, but should not be routinely used for the follow-up of other patients [3]. The use of Dx-WBS in lowrisk patients is generally discouraged if serum $\mathrm{Tg}$ is undetectable and neck ultrasound is negative at the first response assessment. However, an analysis by Gonzalez-Carvalho et al. of a large series of patients followed for up to 25 years suggested that routine Dx-WBS is useful at the first followup (6-12 months after RaIT) in all cases [58]. Moreover, there is agreement on the relevant role of Dx-WBS in patients who are positive for $\mathrm{TgAb}$ [59], or with extrathyroid uptake at post-therapy-WBS (pT-WBS) or large thyroid remnants precluding pT-WBS, and in selected cases based on individual risk profiles (e.g., isthmus location of malignant nodule). To date, the use of rhTSH and hybrid SPECT/CT has been shown to reduce patient discomfort and significantly improve the diagnostic performance of Dx-WBS using either ${ }^{131} \mathrm{I}$ or ${ }^{123} \mathrm{I}[60-62]$.

${ }^{18} \mathrm{~F}$-FDG-PET/CT is the recommended first-line diagnostic procedure for anaplastic and other aggressive thyroid cancers, and is an important second-line procedure for DTC follow-up [3]. In the latter setting, ${ }^{18} \mathrm{~F}$-FDG-PET/CT is especially recommended in patients with increasing $\mathrm{Tg}$ levels, negative ultrasound, and RAI imaging results [3]. Serum Tg thresholds used to select patients for PET/CT imaging vary widely in literature and should be optimized locally, taking into account patient characteristics and the serum $\mathrm{Tg}$ and $\mathrm{TgAb}$ assays used. Interestingly, evaluation of $\mathrm{Tg}$ doubling time can be used in addition to ${ }^{18} \mathrm{~F}$-FDGPET/CT (Fig. 3) [63]. Current data also suggest a role for ${ }^{18} \mathrm{~F}$-FDG-PET/CT in patients with negative ultrasound and Dx-WBS who have increasing TgAb levels [64-66]. In addition, ${ }^{18}$ F-FDG-PET-CT is advised in patients with metastatic DTC, poorly DTC histotypes; to identify patients 
at highest risk for rapid progression and for evaluating the response to systemic and/or local treatments $[1,3]$.

\section{Key points and practical indications}

- Follow-up after primary treatment is currently based on dynamic risk stratification, with neck ultrasound and measurement of $\mathrm{Tg}$ levels (either unstimulated or after TSH stimulation) being standard.

- Dx-WBS is especially suggested in patients with:

- intermediate- or high-risk DTC;

- extra-thyroid RAI uptake on pT-WBS;

- large thyroid remnants reducing reliability of pTWBS;

- positive $\operatorname{TgAb}$, limiting the validity of $\mathrm{Tg}$ as a DTC tumor marker.

- ${ }^{18}$ F-FDG-PET-CT should be considered for patients with negative post-treatment WBS or diagnostic WBS and neck ultrasound despite:

- elevated basal or stimulated serum Tg levels ( $>5.5 \mathrm{ng} /$ $\mathrm{ml}$ or $>10 \mathrm{ng} / \mathrm{ml}$, respectively) or serum Tg doubling time is $<1$ year (regardless of initial value);

- increasing $\mathrm{TgAb}$.

- In addition, 18F-FDG-PET-CT should be always considered:

- in patients with poorly differentiated thyroid cancer;

- in patients with metastatic disease to identify those at highest risk for rapid disease progression;

- for evaluating the response to systemic and/or local treatments in patients with advanced disease.

\section{Metastatic DTC patients}

Loco-regional and distant metastases can be detected at diagnosis, during postoperative assessment with ${ }^{123 / 131} \mathrm{I}$ RAI Dx-WBS, following RaIT, or during follow-up [3, 67]. Distant metastases are more frequent in patients with highrisk DTC [59]; however, they can also be found in patients with low-risk DTC $[68,69]$. A summary of the risk factors associated with a worse prognosis in metastatic DTC is shown in Table 2 [70].

Management of metastatic DTC should involve a multidisciplinary team and should be based both on local and systemic treatments $[3,71,72]$. In patients with RAI-avid metastatic disease, RaIT remains the treatment of choice [3], providing a favorable impact on overall survival and disease-free survival [73-76]. The administered RAI activity can be determined empirically or based on a dosimetric approach $[72,77,78]$. The empiric approach uses a fixed activity (i.e., 3.7-11.1 GBq), which is selected based on disease stage, age, burden of disease, and kidney function [61]. In addition, important limiting factors such as
Table 2 Factors associated with worse prognosis in patients with metastatic differentiated thyroid carcinoma

\begin{tabular}{l}
\hline Risk factors \\
\hline Age $>55$ years \\
Male gender \\
Follicular histology \\
Distant metastases at diagnosis \\
Bone or combined distant metastases (e.g., bone and lung \\
metastases) \\
RaIT-refractory disease
\end{tabular}

RaIT radioiodine therapy

reduced bone marrow reserve (mainly in patients $>70$ years) and hampered lung function (especially in patients with disseminated lung metastases and in pediatric patients) should also be taken into account when choosing empiric activity [72]. This approach is simple, effective, and widely used in clinical practice. The potential limitations include over- or under-treatment and a hypothetical progressive loss of efficacy after repeated treatment [59, 72, 78-80].

The dosimetric approach uses patient-tailored activities according to the principles of As High As Safely Administrable [81] and/or As Low As Reasonably Achievable (ALARA) [34]. This approach is complex to implement, but offers a number of important theoretical advantages, including high rates of therapeutic success with a single treatment, the possibility of adjusting the radiation activity to the target volume, and increased safety for nontarget organs and tissues. Currently, due to a lack of consistent evidence, it is challenging to confirm a preference for the empiric or dosimetric strategy for RaIT [3]. However, the latter approach may be preferable in special conditions (e.g., renal failure, diffuse miliary lung metastases, and reduced bone marrow reserve) in order to reduce toxicity [82-85].

To date, regardless of the strategy used, THW is indicated in patients preparing for RaIT [3], with the use of rhTSH reserved for patients whose endogenous TSH stimulation is precluded (e.g., pituitary diseases) or clinically contraindicated [2, 4, 72, 86-88]. Note, if the dosimetric approach is chosen, RaIT must be performed in the same functional status (i.e., hypothyroidism or euthyroidism) that was used for dosimetric calculations.

In patients with RAI-avid metastatic DTC, RaIT should be repeated until a complete response is achieved (i.e., no abnormal RAI uptake at Dx- or pT-WBS and stimulated Tg levels below the functional sensitivity cut-off in absence of $\mathrm{TgAb}$ ) or there is an adequate and durable response (i.e., slow or no progression of disease) as previously described [89, 90].

Conversely, RaIT should be stopped in patients with unresponsive, RaIT-refractory disease (Table 3) [72, 90]. In this light, some criteria were proposed in literature (e.g., negative RAI imaging, cumulative activity 
Table 3 Definition and initial management of radioiodine therapyrefractory differentiated thyroid carcinoma

Recommendations

- A negative Dx-WBS or pT-WBS is not sufficient to classify a patient as RaIT-refractory.

- The quality of the various assessments performed should always be carefully checked.

- Patients with $\geq 1$ negative lesion on Dx-WBS should not be considered refractory but should receive local treatment for WBSnegative lesions and RaIT for RAI-avid lesions.

- Assessment of structural response to treatment should not strictly adhere to the RECIST criteria but should be individualized by taking into account patient clinical status and wishes.

- The overall course of serum Tg levels should be evaluated, not absolute Tg levels.

- The duration of response to treatment should be recorded ( $<6$ months or $>12$ months, or in between).

- The overall amount of ${ }^{131}$ I-RAI activity administered should be monitored.

- The frequency and severity of adverse events should be recorded.

- The cumulative administered ${ }^{131}$ I-RAI activity being above the suggested limit is not sufficient to define a patient as having RaITrefractory disease.

$D x$-WBS diagnostic WBS, $p T$-WBS post-therapy WBS, RAI radioiodine, RaIT radioiodine therapy, RECIST Response Evaluation Criteria In Solid Tumors, $T g$ thyroglobulin, WBS whole-body scan

$>22.2 \mathrm{GBq})[91,92]$. However, none of the proposed criteria is considered "sacrosanct" [90] and a comprehensive assessment that integrates all of the clinical, biochemical, and imaging data collected during patient management and follow-up should be done before stopping RaIT [90, 93].

\section{Key points and practical indications}

- Management of metastatic DTC should involve a multidisciplinary team and should be based on both local therapy (e.g., surgical resection, radiofrequency ablation, cryoablation, and external beam radiation therapy) and systemic treatment (e.g., RaIT, levothyroxine suppressive therapy, targeted therapy, and cytotoxic chemotherapy).

- In patients with RAI-avid metastatic disease, RaIT may improve overall and disease-free survival and remains the treatment of choice.

- RAI activity to be administered can be determined empirically (3.7-11.1 GBq) or based on a dosimetric (individualized) approach; individualized dosimetry is preferable in selected cases (e.g., renal failure, diffuse miliariform lung metastases, reduced bone marrow reserve, and pediatric patients).

- THW is recommended to prepare patients with metastatic DTC for RaIT; however, rhTSH is mandatory when endogenous stimulation is precluded (i.e., pituitary diseases) or clinically contraindicated.

- The definition of RaIT-refractory patients (Table 3) is evolving toward an individualized and dynamic definition that can integrate all of the clinical, biochemical, and instrumental data collected during patient management and follow-up.

\section{Conclusions}

The Martinique Principles should be implemented in routine clinical practice in order to optimize clinical management/ outcomes in patients with DTC. Based on the clinical experience of five thyroid cancer experts, we provide a suggested approach for assessing and diagnosing thyroid nodules in clinical practice, as well as our recommendations for the use of surgery and postoperative RaIT, and longterm follow-up of patients with DTC. The multidisciplinary approach is particularly important in the management of patients with metastatic DTC, which should be individualized according to disease status and comorbidities. Comprehensive assessment of patients with suspected RaITrefractory disease is recommended before stopping RaIT.

Acknowledgements The authors would like to thank Sarah Greig, $\mathrm{PhD}$, of Springer Healthcare Communications, who provided medical writing support funded by Sanofi.

\section{Compliance with ethical standards}

Conflict of interest A.C. has participated in the speaker program and acted as an advisor for Sanofi Genzyme. D.B. and M.G. have no conflicts of interest to declare. F.C. has acted as an advisor for QB Group Srl. L.G. has participated in the speaker program and acted as an advisor for Roche Diagnostics and Sanofi Genzyme, and has participated in the speaker program for IBSA SA and BRAHMS GmbH.

Ethical approval This article does not contain any studies with animals or human participants performed by any of the authors.

Publisher's note Springer Nature remains neutral with regard to jurisdictional claims in published maps and institutional affiliations.

Open Access This article is licensed under a Creative Commons Attribution 4.0 International License, which permits use, sharing, adaptation, distribution and reproduction in any medium or format, as long as you give appropriate credit to the original author(s) and the source, provide a link to the Creative Commons license, and indicate if changes were made. The images or other third party material in this article are included in the article's Creative Commons license, unless indicated otherwise in a credit line to the material. If material is not included in the article's Creative Commons license and your intended use is not permitted by statutory regulation or exceeds the permitted use, you will need to obtain permission directly from the copyright holder. To view a copy of this license, visit http://creativecommons. org/licenses/by/4.0/. 


\section{References}

1. L. Lamartina, G. Grani, C. Durante, I. Borget, S. Filetti, M. Schlumberger, Follow-up of differentiated thyroid cancer - what should (and what should not) be done. Nat. Rev. Endocrinol. 14 (9), 538-551 (2018)

2. L. Giovanella, L.H. Duntas, Management of endocrine disease: the role of rhTSH in the management of differentiated thyroid cancer: pros and cons. Eur. J. Endocrinol. 181(4), R133-R145 (2019)

3. B.R. Haugen, E.K. Alexander, K.C. Bible, G.M. Doherty, S.J. Mandel, Y.E. Nikiforov, F. Pacini, G.W. Randolph, A.M. Sawka, M. Schlumberger, K.G. Schuff, S.I. Sherman, J.A. Sosa, D.L. Steward, R.M. Tuttle, L. Wartofsky, 2015 American Thyroid Association management guidelines for adult patients with thyroid nodules and differentiated thyroid cancer: the American Thyroid Association Guidelines Task Force on thyroid nodules and differentiated thyroid cancer. Thyroid 26(1), 1-133 (2016)

4. M. Luster, S.E. Clarke, M. Dietlein, M. Lassmann, P. Lind, W.J. Oyen, J. Tennvall, E. Bombardieri, M. European Association of Nuclear, guidelines for radioiodine therapy of differentiated thyroid cancer. Eur. J. Nucl. Med. Mol. Imaging 35(10), 1941-1959 (2008)

5. O. Maas, F. Forrer, M. Maas, C.M. Panje, J. Blautzik, M. Bruhlmeier, I. Engel-Bicik, L. Giovanella, A. Haldemann, M.E. Kamel, S. Kneifel, C. Rottenburger, N. Schaefer, M.A. Walter, S. Weidner, P.M. Putora, Variations in radioiodine ablation: decision-making after total thyroidectomy. Eur. J. Nucl. Med. Mol. Imaging 47(3), 554-560 (2020)

6. R.M. Tuttle, S. Ahuja, A.M. Avram, V.J. Bernet, P. Bourguet, G. H. Daniels, G. Dillehay, C. Draganescu, G. Flux, D. Fuhrer, L. Giovanella, B. Greenspan, M. Luster, K. Muylle, J.W.A. Smit, D. Van Nostrand, F.A. Verburg, L. Hegedus, Controversies, consensus, and collaboration in the use of ${ }^{131}$ I therapy in differentiated thyroid cancer: a joint statement from the American Thyroid Association, the European Association of Nuclear Medicine, the Society of Nuclear Medicine and Molecular Imaging, and the European Thyroid Association. Thyroid 29(4), 461-470 (2019)

7. L. Giovanella, F. D'Aurizio, A. Campenní, R.M. Ruggeri, S. Baldari, F.A. Verburg, P. Trimboli, L. Ceriani, Searching for the most effective thyrotropin (TSH) threshold to rule-out autonomously functioning thyroid nodules in iodine deficient regions. Endocrine 54(3), 757-761 (2016)

8. L. Giovanella, A.M. Avram, I. Iakovou, J. Kwak, S.A. Lawson, E. Lulaj, M. Luster, A. Piccardo, M. Schmidt, M. Tulchinsky, F.A. Verburg, E. Wolin, EANM practice guideline/SNMMI procedure standard for RAIU and thyroid scintigraphy. Eur. J. Nucl. Med. Mol. Imaging 46(12), 2514-2525 (2019)

9. L. Giovanella, L. Ceriani, G. Treglia, Role of isotope scan, including positron emission tomography/computed tomography, in nodular goitre. Best Pract. Res. Clin. Endocrinol. Metab. 28(4), 507-518 (2014)

10. M. Castellana, C. Castellana, G. Treglia, F. Giorgino, L. Giovanella, G. Russ, P. Trimboli, Performance of five ultrasound risk stratification systems in selecting thyroid nodules for FNA. A meta-analysis. J. Clin. Endocrinol. Metab. (2019). https://doi.org/ 10.1210/clinem/dgz170

11. F. Nardi, F. Basolo, A. Crescenzi, G. Fadda, A. Frasoldati, F. Orlandi, L. Palombini, E. Papini, M. Zini, A. Pontecorvi, P. Vitti, Italian consensus for the classification and reporting of thyroid cytology. J. Endocrinol. Investig. 37(6), 593-599 (2014)

12. S.P. Hodak, D.S. Rosenthal, C. American Thyroid Association Clinical Affairs, Information for clinicians: commercially available molecular diagnosis testing in the evaluation of thyroid nodule fineneedle aspiration specimens. Thyroid 23(2), 131-134 (2013)
13. Y.E. Nikiforov, N.P. Ohori, S.P. Hodak, S.E. Carty, S.O. LeBeau, R.L. Ferris, L. Yip, R.R. Seethala, M.E. Tublin, M.T. Stang, C. Coyne, J.T. Johnson, A.F. Stewart, M.N. Nikiforova, Impact of mutational testing on the diagnosis and management of patients with cytologically indeterminate thyroid nodules: a prospective analysis of 1056 FNA samples. J. Clin. Endocrinol. Metab. 96 (11), 3390-3397 (2011)

14. A. Campenní, L. Giovanella, M. Siracusa, A. Alibrandi, S.A. Pignata, S. Giovinazzo, F. Trimarchi, R.M. Ruggeri, S. Baldari, ${ }^{99 m}$ Tc-Methoxy-isobutyl-isonitrile scintigraphy is a useful tool for assessing the risk of malignancy in thyroid nodules with indeterminate fine-needle cytology. Thyroid 26(8), 1101-1109 (2016)

15. A. Campenní, M. Siracusa, R.M. Ruggeri, R. Laudicella, S.A. Pignata, S. Baldari, L. Giovanella, Differentiating malignant from benign thyroid nodules with indeterminate cytology by $(99 \mathrm{~m}) \mathrm{Tc}-$ MIBI scan: a new quantitative method for improving diagnostic accuracy. Sci. Rep. 7(1), 6147 (2017)

16. A. Campenní, P. Trimboli, L. Giovanella, Re: "Diagnostic performance of technetium-99m methoxy-isobutyl-isonitrile for differentiation of malignant thyroid nodules: a systematic review and meta-analysis" by Kim et al. (Thyroid 2018;28:1339-1348). Thyroid 29(6), 896-897 (2019)

17. L. Giovanella, A. Campenní, G. Treglia, F.A. Verburg, P. Trimboli, L. Ceriani, M. Bongiovanni, Molecular imaging with ${ }^{99 \mathrm{~m}} \mathrm{Tc}-$ MIBI and molecular testing for mutations in differentiating benign from malignant follicular neoplasm: a prospective comparison. Eur. J. Nucl. Med. Mol. Imaging 43(6), 1018-1026 (2016)

18. E.J. de Koster, L.F. de Geus-Oei, O.M. Dekkers, I. van Engen-van Grunsven, J. Hamming, E.P.M. Corssmit, H. Morreau, A. Schepers, J. Smit, W.J.G. Oyen, D. Vriens, Diagnostic utility of molecular and imaging biomarkers in cytological indeterminate thyroid nodules. Endocr. Rev. 39(2), 154-191 (2018)

19. K.T. Robbins, A.R. Shaha, J.E. Medina, J.A. Califano, G.T. Wolf, A. Ferlito, P.M. Som, T.A. Day; A.H. Committee for Neck Dissection Classification, S. Neck, Consensus statement on the classification and terminology of neck dissection. Arch. Otolaryngol. Head Neck Surg. 134(5), 536-538 (2008)

20. A. Campenní, L. Giovanella, M. Siracusa, M.E. Stipo, A. Alibrandi, M. Cucinotta, R.M. Ruggeri, S. Baldari, Is malignant nodule topography an additional risk factor for metastatic disease in low-risk differentiated thyroid cancer? Thyroid 24(11), 1607-1611 (2014)

21. I.J. Nixon, F.L. Palmer, M.M. Whitcher, A.R. Shaha, J.P. Shah, S. G. Patel, I. Ganly, Thyroid isthmusectomy for well-differentiated thyroid cancer. Ann. Surg. Oncol. 18(3), 767-770 (2011)

22. I. Vasileiadis, G. Boutzios, M. Karalaki, E. Misiakos, T. Karatzas, Papillary thyroid carcinoma of the isthmus: total thyroidectomy or isthmusectomy? Am. J. Surg. 216(1), 135-139 (2018)

23. Y. Geron, C. Benbassat, M. Shteinshneider, S. Koren, K. Or, E. Markus, D. Hirsch, L.M. Kalmovich, Long-term outcome after hemithyroidectomy for papillary thyroid cancer: a comparative study and review of the literature. Cancers 11(1), E26 (2018)

24. A. Machens, H.J. Holzhausen, H. Dralle, The prognostic value of primary tumor size in papillary and follicular thyroid carcinoma. Cancer 103(11), 2269-2273 (2005)

25. S.R. Rajjoub, H. Yan, N.A. Calcatera, K. Kuchta, C.E. Wang, W. Lutfi, T.A. Moo-Young, D.J. Winchester, R.A. Prinz, Thyroid lobectomy is not sufficient for T2 papillary thyroid cancers. Surgery 163(5), 1134-1143 (2018)

26. H. Zulewski, L. Giovanella, S. Bilz, E. Christ, A. Haldemann, H. Steinert, S. Weidner, D. Oertli, F. Triponez, T. Clerici, A. Minder, M. Dettmer, P. Komminoth, Multidisciplinary approach for riskoriented treatment of low-risk papillary thyroid cancer in Switzerland. Swiss Med. Wkly. 149, w14700 (2019) 
27. D.S. Cooper, G.M. Doherty, B.R. Haugen, R.T. Kloos, S.L. Lee, S.J. Mandel, E.L. Mazzaferri, B. McIver, F. Pacini, M. Schlumberger, S.I. Sherman, D.L. Steward, R.M. Tuttle, Revised American Thyroid Association management guidelines for patients with thyroid nodules and differentiated thyroid cancer. Thyroid 19(11), 1167-1214 (2009)

28. M. B. Amin, S. Edge, F. Greene, D. R. Byrd, R. K. Brookland, M. $\mathrm{K}$. Washington, et al. (eds), AJCC cancer staging manual, 8th edn. (Springer International Publishing, Switzerland, 2017)

29. K.C. Loh, F.S. Greenspan, L. Gee, T.R. Miller, P.P. Yeo, Pathological tumor-node-metastasis (pTNM) staging for papillary and follicular thyroid carcinomas: a retrospective analysis of 700 patients. J. Clin. Endocrinol. Metab. 82(11), 3553-3562 (1997)

30. E.L. Mazzaferri, R.T. Kloos, Clinical review 128: current approaches to primary therapy for papillary and follicular thyroid cancer. J. Clin. Endocrinol. Metab. 86(4), 1447-1463 (2001)

31. N.A. Samaan, P.N. Schultz, R.C. Hickey, H. Goepfert, T.P. Haynie, D.A. Johnston, N.G. Ordonez, The results of various modalities of treatment of well differentiated thyroid carcinomas: a retrospective review of 1599 patients. J. Clin. Endocrinol. Metab. 75(3), 714-720 (1992)

32. A.M. Sawka, K. Thephamongkhol, M. Brouwers, L. Thabane, G. Browman, H.C. Gerstein, Clinical review 170: a systematic review and metaanalysis of the effectiveness of radioactive iodine remnant ablation for well-differentiated thyroid cancer. J. Clin. Endocrinol. Metab. 89(8), 3668-3676 (2004)

33. J.H. Chung, Low iodine diet for preparation for radioactive iodine therapy in differentiated thyroid carcinoma in Korea. Endocrinol. Metab. (Seoul) 28(3), 157-163 (2013)

34. H.R. Maxon, S.R. Thomas, A. Boehringer, J. Drilling, M.I. Sperling, J.C. Sparks, I.W. Chen, Low iodine diet in I-131 ablation of thyroid remnants. Clin. Nucl. Med. 8(3), 123-126 (1983)

35. J.T. Park 2nd, J.V. Hennessey, Two-week low iodine diet is necessary for adequate outpatient preparation for radioiodine rhTSH scanning in patients taking levothyroxine. Thyroid 14(1), 57-63 (2004)

36. C. Tomoda, T. Uruno, Y. Takamura, Y. Ito, A. Miya, K. Kobayashi, F. Matsuzuka, N. Amino, K. Kuma, A. Miyauchi, Reevaluation of stringent low iodine diet in outpatient preparation for radioiodine examination and therapy. Endocr. J. 52(2), 237-240 (2005)

37. D. Barbaro, F.A. Verburg, M. Luster, C. Reiners, D. Rubello, ALARA in rhTSH-stimulated post-surgical thyroid remnant ablation: what is the lowest reasonably achievable activity? Eur. J. Nucl. Med. Mol. Imaging 37(7), 1251-1254 (2010)

38. S.Y. Sohn, J.Y. Choi, H.W. Jang, H.J. Kim, S.M. Jin, S.W. Kim, S. Suh, K.Y. Hur, J.H. Kim, J.H. Chung, S.W. Kim, Association between excessive urinary iodine excretion and failure of radioactive iodine thyroid ablation in patients with papillary thyroid cancer. Thyroid 23(6), 741-747 (2013)

39. A. Campenni, L. Giovanella, S.A. Pignata, M.A. Violi, M. Siracusa, A. Alibrandi, M. Moleti, E. Amato, R.M. Ruggeri, F. Vermiglio, S. Baldari, Thyroid remnant ablation in differentiated thyroid cancer: searching for the most effective radioiodine activity and stimulation strategy in a real-life scenario. Nucl. Med. Commun. 36(11), 1100-1106 (2015)

40. W. Cheng, C. Ma, H. Fu, J. Li, S. Chen, S. Wu, H. Wang, Low- or high-dose radioiodine remnant ablation for differentiated thyroid carcinoma: a meta-analysis. J. Clin. Endocrinol. Metab. 98(4), 1353-1360 (2013)

41. P. Du, X. Jiao, Y. Zhou, Y. Li, S. Kang, D. Zhang, J. Zhang, L. Lv, R. Patel, Low versus high radioiodine activity to ablate the thyroid after thyroidectomy for cancer: a meta-analysis of randomized controlled trials. Endocrine 48(1), 96-105 (2015)
42. B. Fallahi, D. Beiki, A. Takavar, A. Fard-Esfahani, K.A. Gilani, M. Saghari, M. Eftekhari, Low versus high radioiodine dose in postoperative ablation of residual thyroid tissue in patients with differentiated thyroid carcinoma: a large randomized clinical trial. Nucl. Med. Commun. 33(3), 275-282 (2012)

43. A. Campenní, E. Amato, R. Laudicella, A. Alibrandi, D. Cardile, S.A. Pignata, F. Trimarchi, R.M. Ruggeri, L. Auditore, S. Baldari, Recombinant human thyrotropin (rhTSH) versus levo-thyroxine withdrawal in radioiodine therapy of differentiated thyroid cancer patients: differences in abdominal absorbed dose. Endocrine $\mathbf{6 5}$ (1), 132-137 (2019)

44. C. Dueren, M. Dietlein, M. Luster, F. Plenzig, R. Steinke, J. Grimm, P. Groth, W. Eichhorn, C. Reiners, The use of thyrogen in the treatment of differentiated thyroid carcinoma: an intraindividual comparison of clinical effects and implications of daily life. Exp. Clin. Endocrinol. Diabetes 118(8), 513-519 (2010)

45. H. Hänscheid, M. Lassmann, M. Luster, S.R. Thomas, F. Pacini, C. Ceccarelli, P.W. Ladenson, R.L. Wahl, M. Schlumberger, M. Ricard, A. Driedger, R.T. Kloos, S.I. Sherman, B.R. Haugen, V. Carriere, C. Corone, C. Reiners, Iodine biokinetics and dosimetry in radioiodine therapy of thyroid cancer: procedures and results of a prospective international controlled study of ablation after rhTSH or hormone withdrawal. J. Nucl. Med. 47(4), 648-654 (2006)

46. S. Tagay, S. Herpertz, M. Langkafel, Y. Erim, L. Freudenberg, N. Schopper, A. Bockisch, W. Senf, R. Gorges, Health-related quality of life, anxiety and depression in thyroid cancer patients under short-term hypothyroidism and TSH-suppressive levothyroxine treatment. Eur. J. Endocrinol. 153(6), 755-763 (2005)

47. R.M. Tuttle, H. Tala, J. Shah, R. Leboeuf, R. Ghossein, M. Gonen, M. Brokhin, G. Omry, J.A. Fagin, A. Shaha, Estimating risk of recurrence in differentiated thyroid cancer after total thyroidectomy and radioactive iodine remnant ablation: using response to therapy variables to modify the initial risk estimates predicted by the new American Thyroid Association staging system. Thyroid 20(12), 1341-1349 (2010)

48. C. Evans, S. Tennant, P. Perros, Serum thyroglobulin in the monitoring of differentiated thyroid cancer. Scand. J. Clin. Lab. Investig. Suppl. 245, S119-S123 (2016)

49. P. Trimboli, V. Zilioli, M. Imperiali, L. Ceriani, L. Giovanella, High-sensitive basal serum thyroglobulin 6-12 months after thyroid ablation is strongly associated with early response to therapy and event-free survival in patients with low-tointermediate risk differentiated thyroid carcinomas. Eur. J. Endocrinol. 176(5), 497-504 (2017)

50. C. Durante, G. Costante, S. Filetti, Differentiated thyroid carcinoma: defining new paradigms for postoperative management. Endocr. Relat. Cancer 20(4), R141-R154 (2013)

51. L. Lamartina, D. Deandreis, C. Durante, S. Filetti, Endocrine tumours: imaging in the follow-up of differentiated thyroid cancer: current evidence and future perspectives for a risk-adapted approach. Eur. J. Endocrinol. 175(5), R185-R202 (2016)

52. P.W. Rosario, A.F. Mineiro Filho, R.X. Lacerda, D.A. dos Santos, M.R. Calsolari, The value of diagnostic whole-body scanning and serum thyroglobulin in the presence of elevated serum thyrotropin during follow-up of anti-thyroglobulin antibody-positive patients with differentiated thyroid carcinoma who appeared to be free of disease after total thyroidectomy and radioactive iodine ablation. Thyroid 22(2), 113-116 (2012)

53. A. Algeciras-Schimnich, Thyroglobulin measurement in the management of patients with differentiated thyroid cancer. Crit. Rev. Clin. Lab. Sci. 55(3), 205-218 (2018)

54. S. Peiling Yang, A.M. Bach, R.M. Tuttle, S.A. Fish, Frequent screening with serial neck ultrasound is more likely to identify false-positive abnormalities than clinically significant disease in the surveillance of intermediate risk papillary thyroid cancer 
patients without suspicious findings on follow-up ultrasound evaluation. J. Clin. Endocrinol. Metab. 100(4), 1561-1567 (2015)

55. F.A. Verburg, U. Mader, L. Giovanella, M. Luster, C. Reiners, Low or undetectable basal thyroglobulin levels obviate the need for neck ultrasound in differentiated thyroid cancer patients after total thyroidectomy and ${ }^{131} \mathrm{I}$ ablation. Thyroid 28(6), 722-728 (2018)

56. S.P. Yang, A.M. Bach, R.M. Tuttle, S.A. Fish, Serial neck ultrasound is more likely to identify false-positive abnormalities than clinically significant disease in low-risk papillary thyroid cancer patients. Endocr. Pract. 21(12), 1372-1379 (2015)

57. A. Campenní, M. Tulchinsky, Should the use of neckultrasonography be reduced during the follow-up of differentiated thyroid cancer patients with undetectable or low (i.e., $<1$ microg/L) thyroglobulin levels and negative thyroglobulin antibody? J. Endocrinol. Investig. 42(1), 105-106 (2019)

58. J.M. Gonzalez Carvalho, D. Gorlich, O. Schober, C. Wenning, B. Riemann, F.A. Verburg, A. Vrachimis, Evaluation of ${ }^{131}$ I scintigraphy and stimulated thyroglobulin levels in the follow up of patients with DTC: a retrospective analysis of 1420 patients. Eur. J. Nucl. Med. Mol. Imaging 44(5), 744-756 (2017)

59. F.A. Verburg, U. Mader, C. Reiners, H. Hanscheid, Long-term survival in differentiated thyroid cancer is worse after low-activity initial post-surgical 131I therapy in both high- and low-risk patients. J. Clin. Endocrinol. Metab. 99(12), 4487-4496 (2014)

60. A.S. Alzahrani, O. AlShaikh, M. Tuli, A. Al-Sugair, R. Alamawi, M.M. Al-Rasheed, Diagnostic value of recombinant human thyrotropin-stimulated ${ }^{123}$ I whole-body scintigraphy in the followup of patients with differentiated thyroid cancer. Clin. Nucl. Med. 37(3), 229-234 (2012)

61. T. Barwick, I. Murray, H. Megadmi, W.M. Drake, P.N. Plowman, S.A. Akker, S.L. Chew, A.B. Grossman, N. Avril, Single photon emission computed tomography (SPECT)/computed tomography using Iodine-123 in patients with differentiated thyroid cancer: additional value over whole body planar imaging and SPECT. Eur. J. Endocrinol. 162(6), 1131-1139 (2010)

62. A. Spanu, M.E. Solinas, F. Chessa, D. Sanna, S. Nuvoli, G. Madeddu, 131I SPECT/CT in the follow-up of differentiated thyroid carcinoma: incremental value versus planar imaging. J. Nucl. Med. 50(2), 184-190 (2009)

63. L. Giovanella, P. Trimboli, F.A. Verburg, G. Treglia, A. Piccardo, L. Foppiani, L. Ceriani, Thyroglobulin levels and thyroglobulin doubling time independently predict a positive 18F-FDG PET/CT scan in patients with biochemical recurrence of differentiated thyroid carcinoma. Eur. J. Nucl. Med. Mol. Imaging 40(6), 874-880 (2013)

64. S. Asa, S.Y. Aksoy, B. Vatankulu, A. Aliyev, L. Uslu, M. Ozhan, S. Sager, M. Halac, K. Sonmezoglu, The role of FDG-PET/CT in differentiated thyroid cancer patients with negative iodine-131 whole-body scan and elevated anti-Tg level. Ann. Nucl. Med. 28 (10), 970-979 (2014)

65. Y. Liu, The role of 18F-FDG PET/CT in the follow-up of welldifferentiated thyroid cancer with negative thyroglobulin but positive and/or elevated antithyroglobulin antibody. Nucl. Med. Commun. 37(6), 577-582 (2016)

66. E. Ozkan, G. Aras, N.O. Kucuk, Correlation of 18F-FDG PET/CT findings with histopathological results in differentiated thyroid cancer patients who have increased thyroglobulin or antithyroglobulin antibody levels and negative 131I whole-body scan results. Clin. Nucl. Med. 38(5), 326-331 (2013)

67. A.M. Avram, Radioiodine scintigraphy with SPECT/CT: an important diagnostic tool for thyroid cancer staging and risk stratification. J. Nucl. Med. 53(5), 754-764 (2012)

68. H. Kim, H.I. Kim, S.W. Kim, J. Jung, M.J. Jeon, W.G. Kim, T.Y. Kim, H.K. Kim, H.C. Kang, J.M. Han, Y.Y. Cho, T.H. Kim, J.H. Chung, Prognosis of differentiated thyroid carcinoma with initial distant metastasis: a multicenter study in Korea. Endocrinol. Metab. (Seoul) 33(2), 287-295 (2018)

69. E. Roti, R. Rossi, G. Trasforini, F. Bertelli, M.R. Ambrosio, L. Busutti, E.N. Pearce, L.E. Braverman, E.C.Degli Uberti, Clinical and histological characteristics of papillary thyroid microcarcinoma: results of a retrospective study in 243 patients. J. Clin. Endocrinol. Metab. 91(6), 2171-2178 (2006)

70. J.J. Ruegemer, I.D. Hay, E.J. Bergstralh, J.J. Ryan, K.P. Offord, C.A. Gorman, Distant metastases in differentiated thyroid carcinoma: a multivariate analysis of prognostic variables. J. Clin. Endocrinol. Metab. 67(3), 501-508 (1988)

71. A. Lorenzoni, A. Capozza, A. Campennì, L. Giovanella, E. Seregni, Multimodal therapy of advanced differentiated thyroid cancer, with emphasis on the role of radioiodine. Clin. Transl. Imaging 7(6), 427-435 (2019)

72. F.A. Verburg, H. Hanscheid, M. Luster, Radioactive iodine (RAI) therapy for metastatic differentiated thyroid cancer. Best Pract. Res. Clin. Endocrinol. Metab. 31(3), 279-290 (2017)

73. D. Handkiewicz-Junak, J. Wloch, J. Roskosz, J. Krajewska, A. Kropinska, L. Pomorski, A. Kukulska, A. Prokurat, Z. Wygoda, B. Jarzab, Total thyroidectomy and adjuvant radioiodine treatment independently decrease locoregional recurrence risk in childhood and adolescent differentiated thyroid cancer. J. Nucl. Med. 48(6), 879-888 (2007)

74. C. Reiners, J. Biko, H. Haenscheid, H. Hebestreit, S. Kirinjuk, O. Baranowski, R.J. Marlowe, E. Demidchik, V. Drozd, Y. Demidchik, Twenty-five years after Chernobyl: outcome of radioiodine treatment in children and adolescents with very high-risk radiation-induced differentiated thyroid carcinoma. J. Clin. Endocrinol. Metab. 98(7), 3039-3048 (2013)

75. E. Ruel, S. Thomas, M. Dinan, J.M. Perkins, S.A. Roman, J.A. Sosa, Adjuvant radioactive iodine therapy is associated with improved survival for patients with intermediate-risk papillary thyroid cancer. J. Clin. Endocrinol. Metab. 100(4), 1529-1536 (2015)

76. J. Tang, D. Kong, Q. Cui, K. Wang, D. Zhang, X. Liao, Y. Gong, $\mathrm{G}$. $\mathrm{Wu}$, The role of radioactive iodine therapy in papillary thyroid cancer: an observational study based on SEER. Onco. Targets Ther. 11, 3551-3560 (2018)

77. A.M. Avram, Y.K. Dewaraja, Thyroid cancer radiotheragnostics: the case for activity adjusted ${ }^{131}$ I therapy. Clin. Transl. Imaging 6 (5), 335-346 (2018)

78. C.M. Hong, B.C. Ahn, Factors associated with dose determination of radioactive iodine therapy for differentiated thyroid cancer. Nucl. Med. Mol. Imaging 52(4), 247-253 (2018)

79. K. Kulkarni, D. Van Nostrand, F. Atkins, M. Aiken, K. Burman, L. Wartofsky, The relative frequency in which empiric dosages of radioiodine would potentially overtreat or undertreat patients who have metastatic well-differentiated thyroid cancer. Thyroid $\mathbf{1 6}$ (10), 1019-1023 (2006)

80. R.M. Tuttle, R. Leboeuf, R.J. Robbins, R. Qualey, K. Pentlow, S. M. Larson, C.Y. Chan, Empiric radioactive iodine dosing regimens frequently exceed maximum tolerated activity levels in elderly patients with thyroid cancer. J. Nucl. Med. 47(10), 1587-1591 (2006)

81. R.S. Benua, N.R. Cicale, M. Sonenberg, R.W. Rawson, The relation of radioiodine dosimetry to results and complications in the treatment of metastatic thyroid cancer. Am. J. Roentgenol. Radium Ther. Nucl. Med. 87, 171-182 (1962)

82. D. Deandreis, C. Rubino, H. Tala, S. Leboulleux, M. Terroir, E. Baudin, S. Larson, J.A. Fagin, M. Schlumberger, R.M. Tuttle, Comparison of empiric versus whole-body/-blood clearance dosimetry-based approach to radioactive iodine treatment in patients with metastases from differentiated thyroid cancer. J. Nucl. Med. 58(5), 717-722 (2017)

83. J. Klubo-Gwiezdzinska, D. Van Nostrand, F. Atkins, K. Burman, J. Jonklaas, M. Mete, L. Wartofsky, Efficacy of dosimetric versus 
empiric prescribed activity of 131 I for therapy of differentiated thyroid cancer. J. Clin. Endocrinol. Metab. 96(10), 3217-3225 (2011)

84. G. Sgouros, H. Song, P.W. Ladenson, R.L. Wahl, Lung toxicity in radioiodine therapy of thyroid carcinoma: development of a doserate method and dosimetric implications of the $80-\mathrm{mCi}$ rule. J. Nucl. Med. 47(12), 1977-1984 (2006)

85. H. Song, B. He, A. Prideaux, Y. Du, E. Frey, W. Kasecamp, P.W. Ladenson, R.L. Wahl, G. Sgouros, Lung dosimetry for radioiodine treatment planning in the case of diffuse lung metastases. J. Nucl. Med. 47(12), 1985-1994 (2006)

86. J. Klubo-Gwiezdzinska, K.D. Burman, D. Van Nostrand, M. Mete, J. Jonklaas, L. Wartofsky, Radioiodine treatment of metastatic thyroid cancer: relative efficacy and side effect profile of preparation by thyroid hormone withdrawal versus recombinant human thyrotropin. Thyroid 22(3), 310-317 (2012)

87. C. Ma, J. Xie, W. Liu, G. Wang, S. Zuo, X. Wang, F. Wu, Recombinant human thyrotropin (rhTSH) aided radioiodine treatment for residual or metastatic differentiated thyroid cancer. Cochrane Database Syst. Rev. 2010(11), CD008302 (2010). https://doi.org/10.1002/14651858.CD008302.pub2

88. H. Tala, R. Robbins, J.A. Fagin, S.M. Larson, R.M. Tuttle, Fiveyear survival is similar in thyroid cancer patients with distant metastases prepared for radioactive iodine therapy with either thyroid hormone withdrawal or recombinant human TSH. J. Clin. Endocrinol. Metab 96(7), 2105-2111 (2011)

89. R.M. Tuttle, M.S. Brose, E. Grande, S.W. Kim, M. Tahara, M. M. Sabra, Novel concepts for initiating multitargeted kinase inhibitors in radioactive iodine refractory differentiated thyroid cancer. Best Pract. Res. Clin. Endocrinol. Metab. 31(3), 295-305 (2017)

90. D. Van Nostrand, Radioiodine refractory differentiated thyroid cancer: time to update the classifications. Thyroid $\mathbf{2 8}(9)$, 1083-1093 (2018)

91. C. Durante, N. Haddy, E. Baudin, S. Leboulleux, D. Hartl, J.P. Travagli, B. Caillou, M. Ricard, J.D. Lumbroso, F. De Vathaire, M. Schlumberger, Long-term outcome of 444 patients with distant metastases from papillary and follicular thyroid carcinoma: benefits and limits of radioiodine therapy. J. Clin. Endocrinol. Metab. 91(8), 2892-2899 (2006)

92. R. Martins-Filho, L.S. Ward, B.J. Amorim, A.O. Santos, M.C. Lima, C.D. Ramos, P.S. Matos, L.V. Assumpcao, E.E. Camargo, E.C. Etchebehere, Cumulative doses of radioiodine in the treatment of differentiated thyroid carcinoma: knowing when to stop. Arq. Bras. Endocrinol. Metabol. 54(9), 807-812 (2010)

93. L. Giovanella, D. van Nostrand, Advanced differentiated thyroid cancer: when to stop radioiodine? Q. J. Nucl. Med. Mol. Imaging 63(3), 267-270 (2019) 